J. DIFFERENTIAL GEOMETRY

48 (1998) 531-555

\title{
TITS GEOMETRY ASSOCIATED WITH 4-DIMENSIONAL CLOSED REAL-ANALYTIC MANIFOLDS OF NONPOSITIVE CURVATURE
}

\author{
CHRISTOPH HUMMEL \& VIKTOR SCHROEDER
}

\begin{abstract}
We investigate the geometry and topology of the Tits boundary associated with 4-dimensional closed, real-analytic manifolds of nonpositive curvature. We show that each homotopically nontrivial component is a union of geometric boundaries of flats in the corresponding Hadamard manifold and this can be used to describe the structure of its maximal dimensional quasi-flats. The homotopically trivial components are intervals of length smaller than $\pi$ and we give a necessary and sufficient criterion for the existence of such intervals of length greater than zero.
\end{abstract}

\section{Introduction}

The Tits boundary $\partial_{T} X=(X(\infty)$, Td $)$ of a complete, simply connected, non positively curved Riemannian manifold $X$ is a metric space which reflects parts of the asymptotic geometry of $X$. In this paper we obtain a description of this space in the case that $X$ is the universal covering of a compact real-analytic Riemannian manifold of nonpositive sectional curvature and dimension $\leq 4$. While the situation in the 2- and 3-dimensional case is quite obvious and easy to describe (compare Section 1), new and interesting phenomena occur in dimension 4 . Roughly speaking, up to dimension 3 the nontrivial components of $\partial_{T} X$

Received December 26, 1996, and, in revised form, October 16, 1997. The first author was supported by the Swiss National Science Foundation.

Keywords and phrases. nonpositive curvature, Tits geometry, higher rank subspaces

1991 Mathematics Subject Classification. 53C20, 53C23, 57M05. 
are completely determined by the structure of the flat subspaces, while in dimension 4 other 'nonstandard' components occur. However, we will show that the nonstandard components are always intervals of length $<\pi$. In particular these components are contractible. It follows that the homotopy type of $\partial_{T} X$ is already determined by the structure of the flat subspaces in $X$. This enables us to apply results of B. Kleiner on the structure of quasi-flats in $X$. In special cases this leads to a complete description of all quasi-flats in $X$.

To describe the results in more detail, let $M=\Sigma \backslash X$ be a compact real-analytic Riemannian manifold of dimension $4, X$ its Riemannian universal covering space. For our description we can assume that $X$ is irreducible, i.e., $X$ does not split as a nontrivial product. The structure of flat subspaces of $X$ was determined in [8] and we recall the main results in Section 1. We say that a geodesic $c: \mathbb{R} \rightarrow X$ has higher rank, if there exists a geodesic $c^{\prime}: \mathbb{R} \rightarrow X$ with $c(\mathbb{R}) \neq c^{\prime}(\mathbb{R})$ which is parallel to $c$, i.e., their Hausdorff distance satisfies $\operatorname{Hd}\left(c(\mathbb{R}), c^{\prime}(\mathbb{R})\right)<\infty$. A submanifold $V \subset X$ is called a higher rank submanifold if $V$ is a complete totally geodesic submanifold of $X$ with the property that every geodesic $c$ in $V$ has a parallel $c^{\prime}$ with $c^{\prime}(\mathbb{R}) \neq c(\mathbb{R})$ in $V$. In our situation there are only the following possible types of higher rank submanifolds, namely:

(i) $V$ is a $k$-flat for $k=2$ or $k=3$, i.e., $V$ is the image of a totally geodesic and isometric embedding $\mathbb{R}^{k} \rightarrow X$.

(ii) $V$ is isometric to $Q \times \mathbb{R}$, where $Q$ is a 2 -dimensional visibility manifold.

A higher rank submanifold $V$ is closed, if $\Sigma_{V}=\{\sigma \in \Sigma \mid \sigma V=V\}$ operates with compact quotient on $V$. We say that a higher rank submanifold $V$ is maximal, if $V$ is not contained properly in a higher rank submanifold $V$. Clearly every 2 -flat $F$ is contained in some (maybe several) maximal higher rank submanifolds.

In [8] it is proved that modulo $\Sigma$, there are only finitely many maximal higher rank submanifolds and all of them are closed. As a consequence the structure of higher rank submanifolds can be recognized in the fundamental group.

In this paper we address the question whether the flat subspaces determine already the whole asymptotic geometry which is reflected in the Tits boundary $\partial_{T} X$. 
We call a connected component of $\partial_{T} X$ standard if it contains a boundary point of a flat and nonstandard if it is not trivial (i.e., is not a single point) and not standard.

The standard components of $\partial_{T} X$ are described in Section 2, and the main result there is that the standard components are built from the geometric boundaries of the maximal higher rank submanifold.

The nonstandard components are investigated in Section 3. It follows from the properties of the Tits metric that a ray representing a point in a nontrivial component is asymptotically arbitrarily close to the flats and hence to the maximal higher rank submanifolds in $X$. If a ray represents a point in a nonstandard component, it is close to a single higher rank submanifold at most on a bounded interval. This gives rise to an infinite sequence of maximal higher rank subspaces associated to such a ray. Up to some initial elements, this sequence is determined by the corresponding nonstandard component (see Proposition 3.4). In particular, each nonstandard component can be encoded by a sequence of maximal higher rank subspaces.

Below we state the main result of the present paper. It is an existence result for nonstandard components in $\partial_{T} X$, and we show that the nonstandard components are always intervals of some length $<\pi$. In particular they are contractible.

Theorem 1. Let $X$ be the Riemannian universal covering space of a 4-dimensional closed, real-analytic manifold of nonpositive curvature. Then the following are true:

(1) Every nonstandard connected component of $\partial_{T} X$ is an interval of length $<\pi-\delta_{0}$ where $\delta_{0}>0$ depends only on $X$.

(2) The Tits boundary $\partial_{T} X$ contains nonstandard connected components if and only if $X$ contains two maximal higher rank submanifolds $W_{1}$ and $W_{2}$ of dimension 3 with $W_{1} \cap W_{2} \neq \varnothing$ and $W_{1} \neq W_{2}$.

Part (1) of this theorem is proved in Section 3, and Part (2) in Section 4.

\section{Remarks.}

1. The connected components of a length space coincide with its path connected components.

2. There are examples of Hadamard manifolds $X$, constructed in [1], satisfying the assumption of the theorem and the condition in part (2). 
3. Investigating the geometric boundary of graphmanifolds, C. Croke and B. Kleiner [4] found, to our knowledge, the first examples of a Tits geometry with nontrivial nonstandard components.

We can use our result to study the structure of quasi-flats $f: \mathbb{R}^{2} \rightarrow X$ in the case that $X$ does not contain a 3 -flat.

A 2-dimensional quasi-flat in $X$ is a map $f: \mathbb{R}^{2} \rightarrow X$ such that there are constants $L \geq 1$ and $C \geq 0$ with the property

$$
L^{-1} d(x, y)-C \leq d(f(x), f(y)) \leq L d(x, y)+C
$$

for all $x, y \in \mathbb{R}^{2}$. In this case $f$ is also called an $(L, C)$-quasi-flat.

In Section 5 we will define the conical quasi-flats in $X$. The image of a conical quasi-flat is a cone over a simple closed Tits geodesics in $\partial_{T} X$ and it is contained in a distance tube around a finite union of flats in $X$.

Then one can prove the following result which depends essentially on the structure theory for quasi-flats of B. Kleiner [6].

Theorem 2. Let $X$ be as in Theorem 1 and assume additionally that $X$ does not contain any 3-flat. Then the image of every $(L, C)$ quasi-flat $\mathbb{R}^{2} \rightarrow X$ lies in finite distance to a conical quasi-flat and thus in finite distance to a finite number of 2-flats. Furthermore, that number can be bounded depending only on $L$, and it is equal to one, provided the constant $L$ is sufficiently close to 1 .

Remark. The reader should compare this result with the structure of quasi-flats in symmetric spaces (see Section 7.2. in [7] and [5]).

We are grateful to the referee who pointed out the following corollary of Theorem 2.

Corollary 3. Let $f: X_{1} \rightarrow X_{2}$ be a quasi-isometry between two manifolds as in Theorem 2. Then $f$ maps 2 -flats to within finite distance of 2-flats.

We would like to thank S. Buyalo, C. Croke and B. Leeb for stimulating discussions, and B. Kleiner also for explaining to us his results on quasi-flats in [6]. The first author is grateful to the Department of Mathematics at the University of Pennsylvania for its hospitality. 


\section{Higher rank submanifolds and discreteness}

In this section we briefly recall the relevant results of [8] and state some discreteness properties. We also fix some of our notation.

In the sequel, $X$ denotes a real-analytic Hadamard manifold, and $\Sigma$ a group acting freely and properly discontinuously by isometries on $X$ such that the quotient $\Sigma \backslash X$ is compact. We let $d$ and $d^{1}$ be the induced distance functions on $X$ and the unit tangent bundle $T^{1} X$ of $X$, respectively. The geometric boundary $X(\infty)$ of $X$ together with the Tits metric $\mathrm{Td}$ is the Tits boundary denoted by $\partial_{T} X$. We write $\angle$ for the Tits angle. For the basic results on the Tits metric we refer to [2] or $[3]$.

If $X$ is reducible, the Tits geometry of $X$ is the spherical join of the Tits geometries of its lower-dimensional, irreducible factors (see 2.3.4 in [7]). For completeness, we describe briefly the structure of $\partial_{T} X$ in the case that $X$ is irreducible and $\operatorname{dim} X<4$ : If $\operatorname{dim} X<3$ then $\partial_{T} X$ is discrete. If $\operatorname{dim} X=3$, then the connected components of $\partial_{T} X$ are points and circles of length $2 \pi$; the circles are precisely the boundaries at infinity of the 2 -flats in $X$ (see [8]).

By the above and under the additional assumption that $X$ is reducible, the statement of Theorem 1 holds trivially and the statement of Theorem 2 follows directly from [6]. Hence we will assume for the rest of the paper that $\operatorname{dim} X=4$ and $X$ is irreducible.

Unless otherwise stated, geodesic segments in $X$ or $\partial_{T} X$ are supposed to be parametrized by arc length. A geodesic ray in $X$ is always parametrized on the interval $[0, \infty)=: \mathbb{R}_{\geq}$. If $c$ is a geodesic segment, $c(\infty)$ and $c(-\infty)$ denote the limit points in $\partial_{T} X$ of the complete extension of $c$ in positive and negative direction, respectively. Suppose $p, q \in X$ and $x, y \in \partial_{T} X$. Then we mean by $\overline{p q}$ the geodesic segment in $X$ connecting $p$ to $q$, and by $\overline{p x}$ the geodesic ray in $X$ starting at $p$ and representing $x$. If $\angle(x, y)<\pi$ we write $\overline{x y}$ for the unique Tits geodesic in $\partial_{T} X$ connecting $x$ to $y$. By $\overrightarrow{p z}$ we denote the unit vector tangent to $\overline{p z}$ at $p$ in the direction of $z \in X \cup X(\infty)$.

If $v \in T^{1} X$ is a unit tangent vector, then $c_{v}: \mathbb{R} \rightarrow X$ is the geodesic with $\dot{c}_{v}(0)=v$. We also write $v( \pm \infty)$ instead of $c_{v}(\infty)$. By $P_{v}$ we denote the parallel set of $c_{v}$, i.e., the subset of $X$ consisting of the union of all unparametrized geodesics parallel to $c_{v}$. By analyticity, $P_{v}$ is either equal to $c_{v}(\mathbb{R})$ or a higher rank subspace of $X$. We recall that a higher rank subspace of $X$ is a totally geodesic submanifold $V$ of $X$ with the property that every geodesic $c$ in $V$ has a parallel $c^{\prime}$ in $V$ with 
$c^{\prime}(\mathbb{R}) \neq c(\mathbb{R})$. The rank of $v \in T^{1} X$ is equal to the dimension of $P_{v}$, and thus there are four possibilities for the rank of a vector $v \in T^{1} X$ :

(i) $\operatorname{rank}(v)=1$, then $c_{v}$ is not contained in a 2 -flat.

(ii) $\operatorname{rank}(v)=2$, then $c_{v}$ is contained in exactly one 2-flat.

(iii) $\operatorname{rank}(v)=3$, then $P_{v}=Q \times \mathbb{R}$ with a complete 2-dimensional factor $Q$.

(iv) $\operatorname{rank}(v)=4$, then $X=P_{v}$, i.e., $X$ is reducible.

We denote the set of unit tangent vectors in $X$ of rank $\geq k$ by

$$
\mathcal{R}_{\geq k}:=\left\{v \in T^{1} X \mid \operatorname{rank}(v) \geq k\right\},
$$

and put $\mathcal{R}_{\geq k}(\infty):=\left\{v(\infty) \mid v \in \mathcal{R}_{\geq k}\right\}$. Since we assumed that $X$ is irreducible, we have that $\mathcal{R}_{\geq 4}=\varnothing$. Then there are three types of higher rank submanifolds: 2-flats, 3 -flats and spaces of the form $W \cong Q \times \mathbb{R}$, where $Q$ is not flat.

We denote by $\mathcal{V}$ the set of all maximal higher rank subspaces of $X$, and by $\mathcal{W} \subset \mathcal{V}$ the subset of all $W$ with $W \cong Q \times \mathbb{R}, Q$ not flat.

For a higher rank subspace $V \in \mathcal{V}$, the boundary $V(\infty)$ is a path connected subset of $\partial_{T} X$. Tits geodesics in $\partial_{T} V$ are also Tits geodesics in $\partial_{T} X$. If $V$ is a 2 - or 3 -flat, then $V(\infty)$ is an $S^{1}$ of length $2 \pi$ or a standard round sphere $S^{2}$, respectively. If $W \cong Q \times \mathbb{R} \in \mathcal{W}$, then $W(\infty) \subset \partial_{T} X$ is a graph with two vertices, and for each point in $Q(\infty)$ an edge of length $\pi$ connecting these two vertices. The union of any two different edges in this graph is a closed Tits geodesic. These closed Tits geodesics are precisely the Tits boundaries of the 2 -flats in $W$. The vertices of this graph are called the singular points of $W(\infty)$ and they are the limit points of the geodesics of rank 3 in $W$.

It is proved in [8] that modulo $\Sigma$ there are only finitely maximal higher rank submanifolds and all of them are closed. We now describe the possible intersection properties of maximal singular submanifolds (compare [8]):

(i) If $F$ is a 3-flat and $V \in \mathcal{V}$ with $V \neq F$, then $F \cap V=\varnothing$.

(ii) If $F \in \mathcal{V}$ is a 2-flat and $F \cap V \neq \varnothing$ for some $V \neq F$ and $V \subset \mathcal{V}$, then $V$ is also a 2-flat and $F \cap V$ is a single point.

(iii) If $W \in \mathcal{W}$ and $W \cap V \neq \varnothing$ for some $V \in \mathcal{V} \backslash\{W\}$, then $V \in \mathcal{W}$ and $W \cap V$ is a 2 -flat. 
Suppose $W_{1}, W_{2} \in \mathcal{W}$ intersect in a 2-flat $F$. This intersection is reflected in $\partial_{T} X$ as follows: $W_{1}(\infty) \cap W_{2}(\infty)=F(\infty)$ and $F(\infty)$ contains both, the singular points of $W_{1}(\infty)$ and $W_{2}(\infty)$. Notice also that the singular points of $W_{1}(\infty)$ do not coincide with the singular points of $W_{2}(\infty)$. We write

$$
\mathcal{F}_{\text {int }}:=\left\{W_{1} \cap W_{2} \mid W_{1}, W_{2} \in \mathcal{W}, \operatorname{dim} W_{1} \cap W_{2}=2\right\}
$$

for the set of intersection flats.

In the following Lemma, we describe the intersection of maximal higher rank subspaces in more detail (see also Lemma 3.4 in [8]).

Lemma 1.1 (Discreteness Lemma). There exist positive constants $D$ and $a$ with $D>2 a$ such that the following holds:

(1) If $V_{1}, V_{2} \in \mathcal{V}$ with $\operatorname{dim} V_{i}=3$ and $d\left(p, V_{i}\right) \leq D$ for $i=1,2$ and some $p \in X$, then $V_{1}, V_{2} \in \mathcal{W}$ and $V_{1} \cap V_{2} \neq \varnothing$.

(2) For $p \in X$ there are at most two maximal higher rank subspaces $V_{1}, V_{2} \in \mathcal{V}$ with $\operatorname{dim} V_{i}=3$ and $d\left(p, V_{i}\right) \leq D$ for $i=1,2$.

(3) Let $v_{1}, v_{2} \in T^{1} X$ with $\operatorname{rank}\left(v_{1}\right)=\operatorname{rank}\left(v_{2}\right)=3$. If $d^{1}\left(v_{1}, v_{2}\right) \leq 2 a$ then $P_{v_{1}}=P_{v_{2}}$.

(4) If $v_{1}, v_{2} \in \mathcal{R}_{\geq 2}$ and $d^{1}\left(v_{1}, v_{2}\right) \leq 2 a$, then $v_{1}, v_{2} \in T V$ for some $V \in \mathcal{V}$ or there are $W_{1}, W_{2} \in \mathcal{W}$ with $v_{1} \in T W_{1}, v_{2} \in T W_{2}$ and $W_{1} \cap W_{2} \neq \varnothing$.

(5) Let $p \in X$ with $d(p, W) \leq$ a for some $W \in \mathcal{W}$. If $d(p, V) \leq$ a for some $V \in \mathcal{V}$ then $V \in \mathcal{W}$ and $V \cap W \neq \varnothing$.

(6) Let $c:[0, \ell] \rightarrow X$ be a geodesic of length $\ell \geq D$. Assume $d(c(t), V) \leq a$ for some $V \in \mathcal{V}$ and for all $t \in[0, \ell]$. Furthermore suppose that $d^{1}\left(\dot{c}(\ell), \mathcal{R}_{\geq 2}\right)<d(c(\ell), V)$; Then there exists some $W \in \mathcal{W}$ with $d(c(\ell), W)<d(c(\ell), V)$. furthermore, $V \in \mathcal{W}$ and $V \cap W \neq \varnothing$.

(7) Let $W_{1}, W_{2} \in \mathcal{W}$ such that $F:=W_{1} \cap W_{2} \in \mathcal{F}_{\text {int }}$. Denote by $z_{i}^{+}, z_{i}^{-}$the two singular points of $W_{i}(\infty), i=1,2$. If $p \in X$ with $d(p, F)=D$ and $d\left(p, W_{1}\right) \leq a$, then $\angle_{p}\left(z_{2}^{+}, z_{2}^{-}\right) \leq \pi-a$.

Proof. Note that (1), (2) and (3) are proved in [8, Lemma 3.6]. We choose $D>0$ such that (1) and (2) are satisfied. We will show that 
for each item $(3), \ldots,(7)$ there are constants $a_{3}, \ldots, a_{7} \in(0, D / 2)$ such that (i) is satisfied with $a=a_{i}$. Finally define $a:=\min \left\{a_{3}, \ldots, a_{7}\right\}$. As already mentioned, the existence of $a_{3}$ follows from [8].

In order to show (4) we assume the contrary. Then there exist sequences $\left(V_{i}\right)_{i \geq 1}$ and $\left(V_{i}^{\prime}\right)_{i \geq 1}$ in $\mathcal{V}$ with $V_{i} \neq V_{i}^{\prime}, v_{i} \in T^{1} V_{i}, v_{i}^{\prime} \in T^{1} V_{i}^{\prime}$ for each $i$ and $\lim _{i \rightarrow \infty} d^{1}\left(v_{i}, v_{i}^{\prime}\right)=0$ such that $V_{i} \notin \mathcal{W}$ or $V_{i} \cap V_{i}^{\prime}=\varnothing$. By the co-compactness of $\Sigma$ we can assume that, after passing to subsequences, $\left(v_{i}\right)_{i \geq 1}$ and $\left(v_{i}^{\prime}\right)_{i \geq 1}$ converge to the same vector $v$. Moreover, by the finiteness of $\mathcal{V}$ modulo $\Sigma$ we can assume that $V_{i}=: V$ and $V_{i}^{\prime}=: V^{\prime}$ are independent of $i$. Consequently, $v \in T V \cap T V^{\prime}$. It follows that $V, V^{\prime} \in \mathcal{W}$ and $V \cap V^{\prime} \neq \varnothing$, a contradiction.

By (iii) above, we can choose $a_{5}>0$ such that $d(W, F)>2 a_{5}$ for each $W \in \mathcal{W}$ and each flat $F \in \mathcal{V}$ since modulo $\Sigma$ there are only finitely many maximal higher rank subspaces of $X$. Now (5) is a consequence of $(1)$.

To prove (6) we choose $a_{6}<a_{4}$ such that the following holds: if $c:[0, \ell] \rightarrow X$ is a geodesic of length $\ell \geq D$ and $d(c(t), V) \leq a_{6}$ for all $t \in[0, \ell]$, then $d^{1}\left(\dot{c}(\ell), T^{1} V\right) \leq a_{4}$. Consider now a geodesic $c:[0, \ell] \rightarrow X$ with $d(c(t), V) \leq a_{6}$ for all $t \in[0, \ell]$ and $d^{1}\left(\dot{c}(\ell), \mathcal{R}_{\geq 2}\right)<d(c(\ell), V)$. Then there is a $V^{\prime} \in \mathcal{V} \backslash\{V\}$ and $v^{\prime} \in T^{1} V^{\prime}$ with $d^{1}\left(\dot{c}(\ell), v^{\prime}\right)<a_{6}$. By the choice of $a_{6}$ there exists $v \in T^{1} V$ with $d^{1}(\dot{c}(\ell), v)<a_{4}$, and thus $d^{1}\left(v^{\prime}, v\right)<a_{4}+a_{6} \leq 2 a_{4}$. Hence by (4) we have that $V, V^{\prime} \in \mathcal{W}$ and $V \cap V^{\prime} \neq \varnothing$.

If (7) were not true, there would exist sequences $\left(W_{i n}\right)_{n \geq 1}, i=1,2$, in $\mathcal{W}$, points $p_{n} \in X$ with $d\left(p_{n}, W_{1 n}\right) \rightarrow 0$ as $n \rightarrow \infty$ and $d\left(p_{n}, F_{n}\right)=D$ with $F_{n}=W_{1 n} \cap W_{2 n}$. Furthermore, $\measuredangle_{p_{n}}\left(z_{2 n}^{+}, z_{2 n}^{-}\right) \rightarrow \pi$ as $n \rightarrow \infty$ where $z_{i n}^{+}$and $z_{i n}^{-}$denote the singular points of $W_{i n}$. Since $X$ is co-compact and by part (2) we can pass to subsequences such that $p_{n}$ converges to some $p \in X$ as $n \rightarrow \infty$ and $W_{i n}=: W_{i}, F_{n}=: F$ are independent of $n$. Moreover, $z_{i n}^{ \pm}$converge in the sphere topology to the respective points $z_{i}^{ \pm} \in W_{i} \cap \mathcal{R}_{3}(\infty)$. Clearly, $d(p, F)=1, p \in W_{1}$ and $\zeta_{p}\left(z_{2}^{+}, z_{2}^{-}\right)=\pi$ and hence we see that $\operatorname{dim} P_{c}=4$ for a geodesic $c$ in $W_{2}$ with $c( \pm \infty)=z_{2}^{ \pm}$. This is a contradiction. q.e.d.

Notion. We call arguments as in the previous proof accumulation arguments.

We let $\Psi: X \rightarrow \mathcal{P}(\mathcal{V})$ be the function from $X$ to the power set of $\mathcal{V}$ given by

$$
\Psi(p):=\{V \in \mathcal{V} \mid d(p, V) \leq a\}
$$


for $p \in X$. We denote by $|\Psi(p)|$ the number of elements in $\Psi(p)$ which is clearly finite.

\section{Standard components}

In this section we investigate the standard connected components of the metric space $\partial_{T} X$. It turns out that these are the components which arise in a natural way from the boundaries at infinity of higher rank submanifolds.

Recall that $x \in \mathcal{R}_{>2}(\infty)$ if $x=c_{v}(\infty)$ with $\operatorname{rank}(v) \geq 2$. Thus $\mathcal{R}_{\geq 2}(\infty)=\bigcup_{V \in \mathcal{V}} V(\infty)$. The main result of this section is

Proposition 2.1. Let $\mathcal{C}$ be a connected component of the Tits boundary $\partial_{T} X$. If $\mathcal{C} \cap \mathcal{R}_{\geq 2}(\infty) \neq \varnothing$ then $\mathcal{C} \subset \mathcal{R}_{\geq 2}(\infty)$.

Actually, this proposition justifies the notion standard component for a connected component $\mathcal{C}$ of the Tits boundary $\partial_{T} X$ with $\mathcal{C} \cap \mathcal{R}_{\geq 2}(\infty) \neq$ $\varnothing$.

The proposition allows us to show that there are only three possible types of standard components in $\partial_{T} X$.

Corollary 2.2. Assume that $\mathcal{C}$ is a standard component of $\partial_{T} X$. Then exactly one of the following statements is true:

(i) $\mathcal{C}=F(\infty)$, where $F \subset X$ is a 2-flat.

(ii) $\mathcal{C}=F(\infty)$, where $F \subset X$ is a 3-flat.

(iii) $\mathcal{C}=\bigcup_{W \in \mathcal{W}^{*}} W(\infty)$ where $\mathcal{W}^{*} \subset \mathcal{W}$ is a subset which is maximal with respect to Property 2.3 stated below.

Property 2.3. If $W, W^{\prime} \in \mathcal{W}^{*}$, then there exists a sequence $W_{1}, \ldots, W_{k}$ in $\mathcal{W}^{*}$ such that $W=W_{1}, W^{\prime}=W_{k}$ and $W_{i} \cap W_{i+1} \neq \varnothing$ for $1 \leq i \leq k-1$.

Remark. It is clear that $\mathcal{W}^{*} \subset \mathcal{W}$ with Property 2.3 is maximal if and only if $\bigcup_{W \in \mathcal{W}^{*}} W$ is a connected component of $\bigcup_{W \in \mathcal{W}} W \subset X$.

We need some preliminary results:

Lemma 2.4. For all $0<\varepsilon<\pi / 2$ there exists some $\eta>0$ such that the following holds: Let $x_{1}, x_{2} \in \partial_{T} X$ with $\varepsilon \leq \angle\left(x_{1}, x_{2}\right) \leq \pi-\varepsilon$, and let $p \in X$ with $\angle\left(x_{1}, x_{2}\right)-\angle_{p}\left(x_{1}, x_{2}\right) \leq \eta$. Then $d^{1}\left(\overrightarrow{p x_{i}}, \mathcal{R}_{\geq 2}\right) \leq \varepsilon$.

Proof. Assume that the statement does not hold for some $\varepsilon \in(0, \pi / 2)$. Then, using the co-compactness of the group $\Sigma$, the 
continuity of $\angle_{p}$ and the semicontinuity of $\angle$, we obtain $p \in X$ and $x_{1}, x_{2} \in \partial_{T} X$ with

$$
\angle\left(x_{1}, x_{2}\right)=\angle_{p}\left(x_{1}, x_{2}\right) \in(0, \pi)
$$

but $d^{1}\left(\overrightarrow{p x_{i}}, \mathcal{R}_{\geq 2}\right) \geq \varepsilon$ for some $i \in\{1,2\}$. However, (2.0.1) implies that the sector spanned by $\overline{p x_{1}}$ and $\overline{p x_{2}}$ is flat and totally geodesic and hence by analyticity a part of a complete flat which implies $\operatorname{rank}\left(\overrightarrow{p x_{i}}\right) \geq 2$, a contradiction. q.e.d.

Lemma 2.5. Let $\xi:[0, b] \rightarrow \partial_{T} X$ be a unit speed geodesic with $0<b<\pi$ and let $c$ be a ray with $c(\infty)=\xi(0)$. Then there exists some $\tau \in \mathbb{R}$ such such that for all points $p=c(t)$ with $t \geq \tau$, all geodesics $c_{s}:=\overline{p \xi(s)}, s \in[0, b]$, and all $u \geq 0$ we have $d^{1}\left(\dot{c}_{s}(u), \mathcal{R}_{\geq 2}\right)<a$.

Proof. Let $\xi$ and $c$ be as in the assumption of the lemma and choose $\varepsilon>0$ small enough, such that

$$
0<2 \varepsilon \leq \angle(\xi(0), \xi(b))=b \leq \pi-2 \varepsilon \text { and } \varepsilon<a .
$$

Choose $\eta$ for $\varepsilon$ as in Lemma 2.4 and $\tau>0$ such that for $p=c(t)$ with $t \geq \tau$

$$
\angle(\xi(0), \xi(b))-\angle_{p}(\xi(0), \xi(b))<\eta .
$$

Then we have for $s \in[0, b]$ that

$$
\begin{aligned}
& \angle(\xi(s), \xi(0))-\angle_{p}(\xi(s), \xi(0))<\eta, \\
& \measuredangle(\xi(s), \xi(b))-\angle_{p}(\xi(s), \xi(b))<\eta .
\end{aligned}
$$

Thus for all $s \in[0, b]$ and all $u \geq 0$ we have

$$
\begin{aligned}
& \angle(\xi(s), \xi(0))-\angle_{c_{s}(u)}(\xi(s), \xi(0))<\eta, \\
& \angle(\xi(s), \xi(b))-\angle_{c_{s}(u)}(\xi(s), \xi(b))<\eta .
\end{aligned}
$$

where $c_{s}=\overline{p \xi(s)}$. Since for all $s \in[0, b]$

$$
\begin{array}{ll} 
& 0<\varepsilon \leq \angle(\xi(0), \xi(s))=s \leq \pi-\varepsilon \\
\text { or } \quad & 0<\varepsilon \leq \angle(\xi(s), \xi(b))=b-s \leq \pi-\varepsilon,
\end{array}
$$

we see by Lemma 2.4 that

$$
d^{1}\left(\dot{c}_{s}(u), \mathcal{R}_{\geq 2}\right) \leq \varepsilon<a
$$

for each $s \in[0, b]$ and each $u \in[0, \infty)$. q.e.d. 
Lemma 2.6. Let $c: \mathbb{R}_{\geq} \rightarrow X$ be a ray with $c(\infty) \in V(\infty)$ for some $V \in \mathcal{V}$. Assume that $\lim _{t \rightarrow \infty} d(c(t), V)>0$. Then there exists some $W \subset \mathcal{W}$ such that $\lim _{t \rightarrow \infty} d(c(t), W)=0$ and $c(\infty)$ is a singular point of $W(\infty)$. Furthermore, $V \in \mathcal{W}$ and $V \cap W \neq \varnothing$.

Proof. We first use the co-compactness of $\Sigma$ to show that

$$
\lim _{t \rightarrow \infty} d^{1}\left(\dot{c}(t), \mathcal{R}_{3}\right)=0
$$

It suffices to show that for any sequence $t_{i} \rightarrow \infty$ and any isometries $\sigma_{i} \in \Sigma$ such that $\frac{d}{d t}\left(\sigma_{i} \circ c\right)\left(t_{i}\right)$ converges, the limit vector has rank 3 . Let $c_{i}: \mathbb{R}_{\geq} \rightarrow X$ be the ray $c_{i}(t)=\sigma_{i} \circ c\left(t_{i}+t\right)$, i.e., $\dot{c}_{i}(0)=\frac{d}{d t}\left(\sigma_{i} \circ c\right)\left(t_{i}\right)$. Consider the convex function

$$
t \mapsto d\left(c_{i}(t), \sigma_{i} V\right)=d\left(c\left(t+t_{i}\right), V\right),
$$

an accumulation ray $\bar{c}$ of $c_{i}$ and an accumulation higher rank submanifold $\bar{V}$ of $\sigma_{i} V$. It follows that $d(\bar{c}(t), \bar{V})$ is a positive constant. Thus the complete geodesic which extends $\bar{c}$ has a parallel in $\bar{V}$, and since $\bar{V}$ itself has higher rank, this geodesic is of rank 3 .

Since $d^{1}\left(\dot{c}(t), \mathcal{R}_{3}\right) \rightarrow 0$ as $t \rightarrow \infty$, we see by part (3) of Lemma 1.1 that there exists some $W:=P_{v} \in \mathcal{W}$ such that $d^{1}\left(\dot{c}(t), \mathcal{R}_{3} \cap T^{1} W\right) \rightarrow 0$ as $t \rightarrow \infty$. Thus $d(c(t), W) \rightarrow 0$ and $c(\infty)$ is a singular point of $W(\infty)$.

q.e.d.

Lemma 2.7. There exists a constant $\varepsilon_{1}>0$ with the following property. Let $x_{1}=c_{v}(\infty) \in \partial_{T} X$ with $\operatorname{rank}(v)=3$, and let $x_{2} \in \partial_{T} X$ with $\angle\left(x_{1}, x_{2}\right) \leq \varepsilon_{1}$. Then $x_{2} \in P_{v}(\infty)$.

Proof. Assume the contrary. Then there are $v_{i} \in \mathcal{R}_{3}$, $x_{i} \in \partial_{T} X \backslash P_{c_{i}}(\infty)$, where $c_{i}:=c_{v_{i}}$, with $\angle\left(c_{i}(\infty), x_{i}\right) \leq 1 / i$ and $i=1,2, \ldots$.

Choose $p_{i} \in P_{c_{i}}$ and let $\bar{c}_{i}$ be the ray $\overline{p_{i} x_{i}}$. Since $x_{i} \notin P_{c_{i}}(\infty)$, there are $t_{i} \in[0, \infty)$ with $d\left(\bar{c}_{i}\left(t_{i}\right), P_{c_{i}}\right)=1$. Note that $t_{i} \rightarrow \infty$ as $i \rightarrow \infty$. Let $q_{i}:=\bar{c}_{i}\left(t_{i}\right)$. Since $\angle\left(c_{i}(\infty), x_{i}\right) \leq 1 / i$ we have

$$
\begin{aligned}
& \alpha_{i}=\measuredangle_{q_{i}}\left(c_{i}(\infty), x_{i}\right) \rightarrow 0, \\
& \beta_{i}=\measuredangle_{q_{i}}\left(c_{i}(-\infty), p_{i}\right) \leq \pi-\measuredangle_{p_{i}}\left(c_{i}(-\infty), q_{i}\right) \leq \frac{1}{i} \rightarrow 0
\end{aligned}
$$

as $i \rightarrow \infty$. We now use a similar accumulation argument as in Lemma 2.6. Choose $\sigma_{i} \in \Sigma$ such that $\sigma_{i} q_{i}$ converges to some $q \in X, \frac{d}{d t}\left(\sigma_{i} \circ \bar{c}_{i}\right)\left(t_{i}\right)$ converges to some $v \in T_{q}^{1} X$, and $\sigma_{i} P_{c_{i}}$ to some $P_{c^{*}}$. Since $\alpha_{i}, \beta_{i} \rightarrow 0$ 
we have $c_{v}(\infty)=c^{*}(\infty)$ and $c_{v}(-\infty)=c^{*}(\infty)$, and hence $c_{v}$ is parallel to $c^{*}$ with $d\left(c_{v}(\cdot), P_{c^{*}}\right) \equiv 1$. Thus $c_{v}$ has rank 4 in contradiction to the irreducibility of $X$. q.e.d.

Lemma 2.8. Let $\xi:[0, b] \rightarrow \partial_{T} X$ be a unit speed Tits geodesic with $0<b<\pi$. Let $\xi(0) \in V(\infty)$ for some $V \in \mathcal{V}$ and $\xi(s) \notin V(\infty)$ for $s>0$. Then there exists some $W \in \mathcal{W} \backslash\{V\}$ such that $\xi(0) \in W(\infty)$ and $\xi(0)$ is a singular point of $W(\infty)$; furthermore, $V \in \mathcal{W}$.

Proof. Consider a ray $c: \mathbb{R}_{\geq} \rightarrow X$ with $c(\infty)=\xi(0)$. If $\lim _{t \rightarrow \infty} d(c(t), V)>0$, the claim follows by Lemma 2.6. Thus we can assume that

$$
\lim _{t \rightarrow \infty} d(c(t), V)=0 .
$$

If $t>0$ is sufficiently large, then for $p=c(t)$ and $c_{s}=\overline{p \xi(s)}$ we have

$$
d(p, V)<a \quad \text { and } \quad d^{1}\left(\dot{c}_{s}(u), \mathcal{R}_{\geq 2}\right)<a
$$

for each $s \in[0, b]$ and each $u \geq 0$. The second inequality follows from Lemma 2.5. This implies that $\left|\Psi\left(c_{s}(u)\right)\right| \geq 1$ for each $s \in[0, b]$ and $u \geq 0$. For $s \in[0, b]$ define

$$
\varphi(s):=\sup \left\{u \geq 0 \mid d\left(c_{s}(u), V\right) \leq a\right\} .
$$

Note that $\varphi(0)=\infty$, and that $\varphi(s)<\infty$ for all $s \in(0, b]$ since $c_{s}(\infty) \notin V(\infty)$. The function $\varphi$ is continuous, since $d(\cdot, V)$ is convex and $d(p, V)<a$. Now fix a small $s>0$ such that $\varphi(s) \geq D$. Note that $V \notin \Psi\left(c_{s}(u)\right)$ for $u>\varphi(s)$, but $V \in \Psi\left(c_{s}(\varphi(s))\right)$. Since

$$
d^{1}\left(\dot{c}_{s}(\varphi(s)), \mathcal{R}_{\geq 2}\right)<a=d\left(c_{s}(\varphi(s)), V\right)
$$

by part (6) of Lemma 1.1 there exists some $W \in \mathcal{W}$ with $W \in \Psi\left(c_{s}(\varphi(s))\right), W \cap V \neq \varnothing$. From the same lemma we also have that $V \in \mathcal{W}$.

By continuity of $\varphi(s)$ and part (1) of Lemma 1.1 we see that $W \in \Psi\left(c_{s}(\varphi(s))\right.$ for all small $s>0$, so that $\xi(0) \in W(\infty)$. Again by the use of Lemma 2.6 we can assume that $\lim _{t \rightarrow \infty} d(c(t), W)=0$ and hence also that $d(p, W)<a$.

We now claim that $\xi\left(\left[0, b_{1}\right]\right) \subset W(\infty)$ for some $b_{1}>0$. If not the same arguments as above will yield $V^{\prime \prime} \in \mathcal{W}$, then $V^{\prime \prime} \neq V, W$ with $\xi(0) \in V^{\prime \prime}(\infty)$ and $\lim _{t \rightarrow \infty} d\left(c(t), V^{\prime \prime}\right)=0$. This is a contradiction to part (1) of Lemma 1.1. 
Now $\left.\xi\right|_{\left[0, b_{1}\right]}:\left[0, b_{1}\right] \rightarrow W(\infty)$ is a geodesic with $\xi(0) \in V(\infty)$. By the discussion in Section 1, $W(\infty) \cap V(\infty)$ consists of two geodesics $\rho_{1}, \rho_{2}$ (of length $\pi$ ) between the singular points of $W(\infty)$. If $\xi(0)$ is an interior point of one of the $\rho_{i}$, then $\xi(s)$ lies on $\rho_{i}$ for small $s$ and hence is contained in $V(\infty) \cap W(\infty)$. This contradiction shows that $\xi(0)$ is a singular point of $W(\infty)$. q.e.d.

Proof of Proposition 2.1 and Corollary 2.2. Let $\mathcal{C}$ be a connected component of $\partial_{T} X$ and assume that $F(\infty) \cap \mathcal{C} \neq \varnothing$ for some flat $F \in \mathcal{V}$. Then $F(\infty) \subset \mathcal{C}$. If there were a point $x_{1} \in \mathcal{C} \backslash F(\infty)$, then there would be a geodesic $\xi:[0, b] \rightarrow \mathcal{C}$ with $\xi(0) \in F(\infty), \xi(b)=x_{1}$ and $\xi(s) \notin F(\infty)$ for all $s>0$. By Lemma 2.8 we get $F \in \mathcal{W}$, a contradiction.

Now let $\mathcal{C}$ be a connected component and $W_{0}(\infty) \cap \mathcal{C} \neq \varnothing$ for some $W_{0} \in \mathcal{W}$. Let $\mathcal{W}^{*}$ be the maximal set with Property 2.3 and $W_{0} \in$ $\mathcal{W}^{*}$. Obviously $\mathcal{C} \subset \bigcup_{W \in \mathcal{W}^{*}} W(\infty)$. We claim equality. We will show the following: if $x_{1} \in W(\infty)$ for some $W \in \mathcal{W}^{*}$ and $x_{2} \in \partial_{T} X$ with $\angle\left(x_{1}, x_{2}\right)<\varepsilon_{1}$ (the constant of Lemma 2.7), then $x_{2} \in W^{\prime}(\infty)$ for some $W^{\prime}$ with $W \cap W^{\prime} \neq \varnothing$. This gives the result. Let $\xi:[0, b] \rightarrow \partial_{T} X$ be the geodesic from $x_{1}$ to $x_{2}$ and let $s_{0}=\sup \{s \in[0, b] \mid \xi(s) \in W\}$. If $s_{0}=b$ we are finished. If not, Lemma 2.8 implies that $\xi\left(s_{0}\right)$ is a singular point of some $W^{\prime}$ with $\mathcal{W}^{\prime} \cap \mathcal{W} \neq \varnothing$. Hence Lemma 2.7 yields that $\xi([0, b]) \subset W^{\prime}(\infty)$. q.e.d.

\section{Diameter estimate for nonstandard components}

In this section we prove part (1) of Theorem 1, i.e., the diameter of each nonstandard component of $\partial_{T} X$ is uniformly smaller than $\pi$ and that nonstandard components are intervals. Recall that we say that a component of $\partial_{T} X$ is nonstandard if it is not standard and not a single point.

To begin with, we state two lemmas which follow immediately from accumulation arguments. We omit the proofs.

Lemma 3.1. For any $\varepsilon>0$ there exists some $\delta>0$ such that the following is true. Assume $F \subset X$ is a 2 -flat, $c_{i}:[0, D] \rightarrow X, i=1,2$, are two geodesic segments parametrized by arc length with $c_{1}(0)=c_{2}(0)=$ : $p, d(p, F)=D$ and $d\left(c_{i}(t), F\right) \leq D$ for each $t \in[0, D]$ and $i=1,2$. If $\angle\left(\dot{c}_{1}(0), \dot{c}_{2}(0)\right)>\pi-\delta$ then $d^{1}\left(\dot{c}_{1}(0), \mathcal{R}_{3}\right)<\varepsilon$.

Lemma 3.2. For each $\varepsilon>0$ there exists some $\delta>0$ such that the following is true: Suppose that $o, p, q, o^{\prime}, p^{\prime}, q^{\prime} \in X$ with $d(o, p) \geq 1$, 
$d(o, q) \geq 1$ and $d\left(o, o^{\prime}\right)<\delta, d\left(p, p^{\prime}\right)<\delta, d\left(q, q^{\prime}\right)<\delta$. Then $\mid \angle_{o}(p, q)-$ $\angle_{o^{\prime}}\left(p^{\prime}, q^{\prime}\right) \mid<\varepsilon$.

For a point $x \in \partial_{T} X$ we denote by $\mathcal{C}(x)$ the connected component of $\partial_{T} X$ containing $x$. By definition and Proposition $2.1, \mathcal{C}(x)$ is standard if and only if $x \in \mathcal{R}_{\geq 2}(\infty)$. Furthermore, from the previous section we see the following: Given a ray $c$ in $\partial_{T} X$, its limit point $c(\infty)$ is contained in a standard component of $\partial_{T} X$ if and only if

$$
\bigcap_{t \geq t_{0}} \Psi \circ c \neq \varnothing
$$

for some $t_{0} \geq 0$. We now investigate the function $\Psi \circ c$ for rays $c$ representing points in nonstandard components.

Lemma 3.3. Suppose that $x \in \partial_{T} X$ and $\mathcal{C}(x)$ is nonstandard. Let $c: \mathbb{R}_{\geq} \rightarrow X$ be a ray representing $x$. Then there exists some $\tau \geq 0$ such that the following are true:

(i) For each $t \geq \tau, \Psi \circ c(\tau) \subset \mathcal{W}$.

(ii) For each $t \geq \tau,|\Psi \circ c(t)| \in\{1,2\}$.

(iii) $\bigcap_{t \geq t_{1}} \Psi \circ c=\varnothing$ for each $t_{1} \geq 0$.

For a ray $c$ as in the lemma, we denote by $\tau(c)$ the minimal $\tau \geq 0$ such that (i) and (ii) in the lemma hold.

Proof. Lemma 2.5 implies in particular that there exists some $\tau \in \mathbb{R}$ such that for $t \geq \tau, d^{1}\left(\dot{c}(t), \mathcal{R}_{\geq 2}\right)<a$ and hence $|\Psi \circ c(t)| \geq 1$ for $t \geq \tau$. Thus there exists some $V \in \mathcal{V}$ with $d^{1}\left(\dot{c}(t), T^{1} V\right)<a$. Since $\mathcal{C}(x)$ is nonstandard, we see that $c(\infty) \notin V(\infty)$ and thus $d(c(t), V) \rightarrow \infty$ as $t \rightarrow \infty$. Hence $\tau_{1}:=\sup \left\{t \geq \tau \mid d^{1}\left(\dot{c}(t), T^{1} V\right)=a\right\}<\infty$ and let $v \in T^{1} V$ with $d^{1}\left(\dot{c}\left(\tau_{1}\right), v\right)=a$. Since $d^{1}\left(\dot{c}\left(\tau_{1}\right), \mathcal{R}_{>2}\right)<a$, there exist some $V^{\prime} \in \mathcal{V} \backslash\{V\}$ and some $v^{\prime} \in T^{1} V^{\prime}$ with $d^{1}\left(\dot{c}\left(\tau_{1}\right), v^{\prime}\right)<a$. By part (4) of Lemma 1.1 we see that $V, V^{\prime} \in \mathcal{W}$.

This shows that $C:=\{t \geq \tau \mid \Psi \circ c(t) \cap \mathcal{W} \neq \varnothing\}$ is not empty. Clearly, $C$ is closed. We claim that it is an open subset of $[\tau, \infty)$. Let $t \in C$ and $W \in \Psi \circ c(t) \cap \mathcal{W}$. Since $t \geq \tau$, there exists some $V \in \mathcal{V}$ with $d(c(t), V)<a$. By part (5) of Lemma 1.1 we have $V \in \mathcal{W}$. Since $V \in \Psi \circ c(s)$ for $s$ in an open neighbourhood of $t$, we see that $C$ is open. Hence we have proved that $\Psi \circ c(t) \cap \mathcal{W} \neq \varnothing$ for each $t \geq \tau$. Now part (5) of Lemma 1.1 implies that $\Psi \circ c(t) \subset \mathcal{W}$. By part (2) of that 
lemma, $|\Psi \circ c(t)| \leq 2$ and by our assumption $x \notin \mathcal{R}_{\geq 2}(\infty)$ we have that $\bigcap_{t \geq t_{1}} \Psi \circ c=\varnothing$ for each $t_{1} \geq 0$. q.e.d.

Under the assumption of Lemma 3.3 we consider the set

$$
A(c):=\{t \in(\tau(c), \infty)|| \Psi \circ c(t) \mid=1\} .
$$

The set $A(c)$ has the following properties:

(i) $A(c)=\bigcup_{i=1}^{\infty}\left(a_{i}, b_{i}\right)$ where $a_{i}<b_{i}-D<b_{i} \leq a_{i+1}$ and $\left.\Psi \circ c\right|_{\left(a_{i}, b_{i}\right)}$ is constant. We denote the value of $\Psi \circ c$ on $\left(a_{i}, b_{i}\right)$ by $\left\{W_{i}^{*}(c)\right\}$.

(ii) There is a unique sequence $i_{1}<i_{2}<\cdots<i_{k}<i_{k+1}<\cdots$ such that $\left\{i \mid W_{i_{n}}^{*}(c)=W_{i}^{*}(c)\right\}=\left\{i \mid i_{n} \leq i<i_{n+1}\right\}$. We define $W_{n}(c):=W_{i_{n}}^{*}(c)$ for each integer $n \geq 1$.

Indeed, the previous lemma implies that $A(c)$ has infinitely many connected components. Moreover, for a connected component $\left(a^{\prime}, b^{\prime}\right)$ we have $\left|\Psi \circ c\left(a^{\prime}\right)\right|=\left|\Psi \circ c\left(b^{\prime}\right)\right|=2$, and thus $b^{\prime}-a^{\prime}>D$ by Lemma 1.1. Let $\left(a_{i}, b_{i}\right), i=1,2, \ldots$, with $a_{i}<a_{i+1}$ be the connected components of $A(c)$. This shows (i). In order to see (ii), suppose that $W_{i}^{*}(c)=W_{i+l}^{*}(c)$ for some $i$ and some $l>0$. Since the function $d\left(W_{i}^{*}(c), \cdot\right)$ is convex on $X, d\left(W_{i}^{*}(c), c(t)\right) \leq a$ for each $t \in\left(a_{j}, b_{j+l}\right)$. Thus $W_{i}^{*}(c)=W_{i+k}^{*}$ for $k=1,2, \ldots, l$.

Let $c$ and $c^{\prime}$ be rays in $X$. We call the corresponding sequences $\left(W_{n}(c)\right)_{n \geq 1}$ and $\left(W_{n}\left(c^{\prime}\right)\right)_{n \geq 1}$ equivalent if there exist $n_{0}, m_{0} \in \mathbb{Z}$ with $n_{0}>-m_{0}$ such that $W_{n}(c)=W_{n+m_{0}}\left(c^{\prime}\right)$ for each $n \geq n_{0}$.

The next proposition states a property of nonstandard components which is of interest itself, but not directly needed for the proof of Theorem 1. For that purpose Lemma 3.5 below is enough.

Proposition 3.4. Let $\mathcal{C}$ be a nonstandard component of $\partial_{T} X$. Let $c$ and $c^{\prime}$ be two rays in $X$ with $c(\infty), c^{\prime}(\infty) \in \mathcal{C}$. Then $\left(W_{n}(c)\right)_{n \geq 1}$ is equivalent to $\left(W_{n}\left(c^{\prime}\right)\right)_{n \geq 1}$.

Proof. In the first step we consider two rays $c$ and $c^{\prime}$ with $c(\infty)=$ $c^{\prime}(\infty)$ we show that the corresponding sequences of higher rank subspaces are equivalent. We parametrize $c$ and $c^{\prime}$ by arc length, and we may clearly assume that $c(0)$ and $c^{\prime}(0)$ lie on the same horosphere around $c(\infty)=c^{\prime}(\infty)$. We first assume that $d\left(c(t), c^{\prime}(t)\right) \rightarrow 0$ as $t \rightarrow \infty$. Let $A(c)=\bigcup_{i=1}^{\infty}\left(a_{i}, b_{i}\right)$ as above with $\left|b_{i}-a_{i}\right| \geq D$. Again an accumulation argument shows that there exists an $\varepsilon>0$ such that the following holds: If $d\left(c\left(b_{i}\right), V\right)=a=d\left(c\left(a_{i}\right), V\right)$ and $\left|b_{i}-a_{i}\right| \geq D$, then 
$d\left(c\left(\left(b_{i}+a_{i}\right) / 2\right), V\right)<a-\varepsilon$. Since finally $d\left(c(t), c^{\prime}(t)\right)<\varepsilon$, we easily see that the corresponding sequences are equivalent.

If we assume that $t \mapsto d\left(c(t), c^{\prime}(t)\right)$ is bounded from below by a positive constant, then "asymptotically" $c$ and $c^{\prime}$ bound a flat strip. More precisely, an accumulation argument shows that for $t$ large enough, $\dot{c}(t)$ and $\dot{c}^{\prime}(t)$ are close to the same higher rank submanifold, i.e., there exists some $\bar{t}>0$ such that

$$
\Psi \circ c(t) \cap \Psi \circ c^{\prime}(t) \neq \varnothing \quad \text { for each } t \geq \bar{t} .
$$

Pick some $k_{0}>0$ such that for each integer $k \geq k_{0}$,

$$
\inf (\Psi \circ c)^{-1}\left\{W_{k}(c)\right\}>\bar{t} .
$$

We claim that for each $k \geq k_{0}$ there exists some integer $l$ such that $W_{k}(c)=W_{l}\left(c^{\prime}\right)$ and $W_{k+1}(c)=W_{l+1}\left(c^{\prime}\right)$ which imply that $\left(W_{n}(c)\right)_{n \geq 1}$ is equivalent to $\left(W_{n}\left(c^{\prime}\right)\right)_{n \geq 1}$.

To prove this claim, we pick any $k \geq k_{0}$ and abbreviate $W_{1}:=W_{k}(c)$ and $W_{2}:=W_{k+1}(c)$. Observe that $(\Psi \circ c)^{-1}\left(\left\{W_{1}, W_{2}\right\}\right)=:\left[t_{1}, t_{2}\right]$ is a closed interval. By (3.0.1) we have that $W_{i} \in \Psi \circ c^{\prime}\left(t_{i}\right)$ for $i=1,2$. Put

$$
t_{+}:=\sup \left\{t \mid W_{1} \in \Psi \circ c^{\prime}(t)\right\}, \quad t_{-}:=\inf \left\{t \mid W_{2} \in \Psi \circ c^{\prime}(t)\right\},
$$

and claim that $t_{-} \leq t_{+}$. Otherwise there exists some $t_{3} \in\left(t_{+}, t_{-}\right) \subset$ $\left(t_{1}, t_{2}\right)$ with $\Psi \circ c^{\prime}\left(t_{3}\right)=\{W\}$ for some $W \in \mathcal{W} \backslash\left\{W_{1}, W_{2}\right\}$. Thus by (3.0.1), $\left\{W, W_{1}, W_{2}\right\} \subset \Psi \circ c\left(t_{3}\right)$ in contradiction to part (2) of Lemma 1.1 .

Therefore, $\Psi \circ c^{\prime}(t)=\left\{W_{1}\right\}$ for each $t<t_{-}$sufficiently close to $t_{-}$ and $\Psi \circ c^{\prime}(t)=\left\{W_{2}\right\}$ for each $t>t_{+}$sufficiently close to $t_{+}$. This proves the above claim.

With the result proved so far, the proposition is now an immediate consequence of (ii) in the next lemma. q.e.d.

Lemma 3.5. Let $\xi:[0, b] \rightarrow \partial_{T} X$ be a Tits geodesic with $0<b<\pi$ which is contained in a nonstandard component. Then there exists a point $p \in X$ such that for the geodesic variation $c_{s}:=\overline{p \xi(s)}, s \in[0, b]$, the following are true:

(i) $\tau\left(c_{s}\right)=0$ for each $s \in[0, b]$.

(ii) For each integer $n \geq 1, W_{n}\left(c_{s}\right)=: W_{n}$ is independent of $s \in[0, b]$.

(iii) $d(p, V) \geq D$ for each $V \in \mathcal{V} \backslash\left\{W_{1}\right\}$. 
Proof. We pick any ray $c$ with $c(\infty)=\xi(0)$. By Lemma 3.3 and part (2) of Lemma 1.1, there exists an arbitrary large $t_{0}$ such that $\left|\Psi \circ c\left(t_{0}\right)\right|=1$ and (iii) is satisfied with $p=c\left(t_{0}\right)$ and $W_{1}=\Psi \circ c\left(t_{0}\right)$.

If $t_{0}$ with these properties is sufficiently large, we see from Lemma 2.5 that $d^{1}\left(\dot{c}_{s}(u), \mathcal{R}_{\geq 2}\right)<a$ for all $s \in[0, b]$ and all $u \geq 0$. Now the proof of Lemma 3.3 shows that $\tau\left(c_{s}\right)=0$ for each $s \in[0, b]$.

With this choice of $p$ it remains to prove (ii). To that end, we consider for each integer $n \geq 1$ the set

$$
I_{n}:=\left\{s \in[0, b] \mid W_{n}\left(c_{s}\right)=W_{n}\left(c_{0}\right)\right\} .
$$

By the above, $I_{1}=[0, b]$. Given $n \geq 2$, we prove by induction that $I_{n}=$ $[0, b]$. By Lemma 1.1, $I_{n}$ and $[0, b] \backslash I_{n}$ are open subsets of $I_{n-1}=[0, b]$. Since $I_{n} \neq \varnothing$ this finishes the proof of the lemma. q.e.d.

After these preparations, we now prove part (1) of Theorem 1.

Proof (Diameter estimate). Arguing indirectly, we assume there exist $x, y \in \partial_{T} X \backslash \mathcal{R}_{\geq 2}(\infty)$ such that

$$
\pi-\delta_{0}<\angle(x, y)<\pi
$$

where we can choose $\delta_{0}$ as small as we wish. With $\xi:=\overline{x y}$ parametrized on $[0,1]$ we choose $p \in X$ according to Lemma 3.5 and such that $\pi-\delta_{0}<\angle_{p}(x, y)$. We let $c_{s}, s \in[0,1]$, and $\left(W_{i}\right)_{i \geq 1}$ be as in the previous lemma. Then we denote by $t_{i}, i=0,1$, the real numbers such that

$$
d\left(c_{i}\left(t_{i}\right), W_{2} \cap W_{3}\right)=D \quad \text { and } \quad \Psi \circ c_{i}\left(t_{i}\right)=\left\{W_{2}\right\} .
$$

By Lemma 3.1 applied to the geodesic segments $\overline{c_{0}\left(t_{0}\right) c_{1}\left(t_{1}\right)},\left.c_{0}\right|_{\left[t_{0}, t_{0}+D\right]}$ and to the flat $W_{2} \cap W_{3}$ we see that $d^{1}\left(\dot{c}_{0}\left(t_{0}\right), v_{0}\right)<\delta_{1}$ for some $v_{0} \in \mathcal{R}_{3}$, and in the same way that $d^{1}\left(\dot{c}_{1}\left(t_{1}\right), v_{1}\right)<\delta_{1}$ for some $v_{1} \in \mathcal{R}_{3}$. Here we can choose $\delta_{1} \in(0, a)$ as small as we wish, provided $\delta_{0}>0$ is sufficiently small. Part (4) of Lemma 1.1 shows that $v_{i} \in T^{1} W_{2}$ for $i=0,1$.

Let $q_{i}$ denote the base point of $v_{i}$. Since clearly $t_{i}>D$, applying Lemma 3.2 yields that

$$
\angle_{p}\left(q_{i}, c_{i}\left(t_{i}\right)\right)<\delta_{2} \quad \text { and } \quad \angle_{q_{i}}\left(p, v_{i}(\infty)\right)>\pi-\delta_{2}
$$

for some $\delta_{2}$ as small as we wish, provided $\delta_{1}$ is small enough. Consequently,

$$
\measuredangle_{p}\left(v_{1}(\infty), v_{2}(\infty)\right) \geq \pi-\delta_{0}-4 \delta_{2},
$$


in contradiction to part (7) of the Discreteness Lemma 1.1 q.e.d.

Proof (Nonstandard components are intervals). Suppose there exist $x_{1}, x_{2}, x_{3} \in X(\infty) \backslash \mathcal{R}_{\geq 2}(\infty)$ with $0<\angle\left(x_{i}, x_{j}\right)<\pi$ for each $i, j=1,2,3$. Pick $p \in X$ and let $c_{i}:=\overline{p x_{i}}$.

By the previous results, there exist a sequence $\left(F_{n}\right)_{n \geq 1} \subset \mathcal{F}_{\text {int }}$ of intersection flats, for each $i=1,2,3$ a sequence $\left(t_{i n}\right)_{n \geq 1}$ in $\mathbb{R}$ and $v_{i n} \in$ $T^{1} F_{n}$ such that

$$
t_{\text {in }} \rightarrow \infty \quad \text { and } \quad d^{1}\left(\dot{c}_{i}\left(t_{i n}\right), v_{i n}\right) \rightarrow 0 \quad \text { as } n \rightarrow \infty
$$

Indeed, $d^{1}\left(\dot{c}_{i}(t), \mathcal{R}_{\geq 2}\right) \rightarrow 0$ as $t \rightarrow \infty$ and any $W_{1}, W_{2} \in \mathcal{W}$ with $W_{1} \cap$ $W_{2} \in \mathcal{F}_{\text {int }}$ intersect orthogonally.

Claim 1. $\lim _{n \rightarrow \infty} v_{i n}(\infty)=x_{i}$ in the sphere topology.

Let $\alpha>0$ and $E_{i}:=\left\{q \in X \mid \angle_{p}\left(q, x_{i}\right) \leq \alpha\right\}$, and let $o_{i n}$ be the base point of $v_{i n}$. Arguing indirectly, we may assume w.l.o.g. that $c_{v_{i n}}\left(\mathbb{R}_{\geq}\right) \not \subset E_{i}$ for some $i$ and each $n$. Thus there exists some $e_{n} \in$ $\partial E_{i} \cap c_{v_{i n}}\left(\mathbb{R}_{\geq}\right)$. Lemma 3.2 implies that $\angle_{o_{i n}}\left(p, e_{n}\right) \rightarrow \pi$ as $n \rightarrow \infty$. Since $\angle_{p}\left(o_{i n}, c\left(t_{i n}\right)\right) \rightarrow 0$, we conclude that the sum of the interior angles of the triangle $\left(p, o_{i n}, e_{n}\right)$ is greater than $\pi$ for each large $n$. This shows Claim 1.

Claim 2. $\lim _{n \rightarrow \infty} \angle\left(v_{i n}(\infty), v_{j n}(\infty)\right)=\angle\left(x_{i}, x_{j}\right)$. 
We put

$$
\begin{array}{ll}
\alpha_{n}:=\angle_{c_{i}\left(t_{i n}\right)}\left(p, c_{j}\left(t_{j n}\right)\right), & \beta_{n}:=\angle_{c_{j}\left(t_{j n}\right)}\left(p, c_{i}\left(t_{i n}\right)\right), \\
\alpha_{n}^{\prime}:=\pi-\angle_{o_{i n}}\left(v_{i n}(\infty), o_{j n}\right), & \beta_{n}^{\prime}:=\pi-\angle_{o_{j n}}\left(v_{j n}(\infty), o_{i n}\right),
\end{array}
$$

as illustrated in Figure 3. Clearly, $\angle\left(x_{i}, x_{j}\right)=\lim _{n \rightarrow \infty}\left(\pi-\alpha_{n}-\beta_{n}\right)$. We conclude from Lemma 3.2 that $\left|\alpha_{n}-\alpha_{n}^{\prime}\right| \rightarrow 0$ and $\left|\beta_{n}-\beta_{n}^{\prime}\right| \rightarrow 0$ as $n \rightarrow \infty$. Since $\pi-\alpha_{n}-\beta_{n} \geq \angle_{p}\left(x_{i}, x_{j}\right)>0$, we have that $\alpha_{n}^{\prime}+\beta_{n}^{\prime}<\pi$ for $n$ large enough. Thus the geodesics $c_{v_{i n}}$ and $c_{v_{j n}}$ are not parallel and intersect at a point $c_{v_{i n}}\left(s_{i n}\right)=c_{v_{j n}}\left(s_{j n}\right)$ with negative $s_{i n}$ and $s_{j n}$. Therefore $\angle\left(v_{i n}(\infty), v_{j n}(\infty)\right)=\pi-\alpha_{n}^{\prime}-\beta_{n}^{\prime}$. Thus Claim 2 holds.

Claims 1 and 2 imply that nonstandard components are intervals. Indeed, assume that $\angle\left(x_{i}, x_{j}\right)<\pi / 2$ for each $i, j$. Then, after renumbering, we have that $\angle\left(x_{1}, x_{2}\right)+\angle\left(x_{2}, x_{3}\right)=\angle\left(x_{1}, x_{3}\right)$ and in particular that $x_{2}$ is contained in the geodesic segment $\overline{x_{1} x_{3}}$. q.e.d.

\section{Existence of nonstandard components}

In this section we show the existence of nonstandard components in the case that $X$ contains intersection flats. This is part (2) of Theorem 1 and it follows from Proposition 4.1 below.

We assume that there exist different higher rank submanifolds $W_{0}, W_{1} \in \mathcal{W}$ with $F_{0}=W_{0} \cap W_{1}$ being a 2-flat. For $\nu=0,1$ we choose one singular point $x_{\nu} \in W_{\nu}(\infty)$. Then $x_{0}, x_{1} \in F_{0}(\infty)$.

Proposition 4.1. Given $\varepsilon>0$, there exists a nonstandard component with diameter larger than $\angle\left(x_{0}, x_{1}\right)-2 \varepsilon$.

Proof. We call a sequence $\left(c_{1}, \ldots, c_{k}\right)$ of geodesic segments

$$
c_{i}:\left[0, t_{i}\right] \rightarrow X
$$

with $c_{i+1}(0)=c_{i}\left(t_{i}\right)$ a polygon. We also consider infinite polygons $\left(c_{1}, c_{2}, \ldots\right)$. For a geodesic segment $c_{i}:\left[0, t_{i}\right] \rightarrow X$ let $\bar{c}_{i}: \mathbb{R} \rightarrow X$ be the complete geodesic with $\left.\bar{c}_{i}\right|_{\left[0, t_{i}\right]}=c_{i}$. For a given $\varepsilon>0$ we will construct a sequence $\left(\sigma_{i}\right)_{i \geq 1}$ in $\Sigma$ and two polygons, the 'even' polygon $\left(c_{0}, c_{2}, c_{4}, \ldots\right)$ and the 'odd' polygon $\left(c_{1}, c_{3}, c_{5}, \ldots\right)$ where $c_{i}:\left[0, t_{i}\right] \rightarrow X$ for $i=0,1,2, \ldots$. The whole construction will satisfy the following conditions (see also Figure 4):

(i) Let $W_{0}, W_{1}$ be as above. Then $\left(\sigma_{i}\right)_{i \geq 1}$ satisfies: $\sigma_{i} W_{i}=W_{i}$ and $\sigma_{i} x_{i}=x_{i}$ where we define inductively $W_{i+1}:=\sigma_{i} W_{i-1}, x_{i+1}:=$ $\sigma_{i} x_{i-1}$ and $F_{i}:=\sigma_{i} F_{i-1}=W_{i} \cap W_{i+1}$. The $W_{i}$ are all distinct. 
Note that the conditions imply that $x_{i}$ is a singular point of $W_{i}(\infty)$, and that $\angle\left(x_{i}, x_{i+1}\right)=\angle\left(x_{0}, x_{1}\right)$ since $\sigma_{i}$ is an isometry.

(ii) We have $t_{0}=t_{1}=1, c_{0}(0)=c_{1}(0)=p \in F_{0}$ and $\bar{c}_{i}(\infty)=x_{i}$ for $i=0,1,2, \ldots$.

(iii) $d\left(c_{i}\left(t_{i}\right), W_{i}\right) \leq \varepsilon$ for $i=1,2,3, \ldots$

(iv) $\operatorname{Hd}\left(c_{*}\left(\left[0, t_{*}\right]\right) \cup c_{*+2}\left(\left[0, t_{*+2}\right]\right) \cup \cdots \cup c_{i-2}\left(\left[0, t_{i-2}\right]\right) \cup \bar{c}_{i}\left(\mathbb{R}_{\geq}\right), \overline{p x_{i}}\right) \leq$ $\left(1-2^{-i}\right) \varepsilon$ for $i=0,1,2, \ldots$ where $*$ is 0 for $i$ even and 1 for $i$ odd.

\section{FIGURE 2}

Let us assume that we have constructed the situation above. We assume w.l.o.g. that $\varepsilon<(1 / 2) \min \left\{a, \measuredangle\left(x_{0}, x_{1}\right)\right\}$. For $i \geq 0$ let $r_{i}$ be the ray $\overline{p x_{i}}$. Since $d\left(c_{i}\left(t_{i}\right), p\right) \rightarrow \infty$ by (iii), we obtain from (iv) that $\left(\dot{r}_{2 i}(0)\right)_{i \geq 1}$ and $\left(\dot{r}_{2 i+1}(0)\right)_{i \geq 1}$ are Cauchy sequences. Let $r^{+}\left(\right.$resp. $\left.r^{-}\right)$ be the limit ray of $r_{0}, r_{2}, r_{4}, \ldots$ (resp. $r_{1}, r_{3}, r_{5}, \ldots$ ). By (iv) we have that

$$
\begin{aligned}
& \operatorname{Hd}\left(\bigcup_{i=0}^{\infty} c_{2 i}\left(\left[0, t_{2 i}\right]\right), r^{+}\left(\mathbb{R}_{\geq}\right)\right) \leq \varepsilon \text { and } \\
& \operatorname{Hd}\left(\bigcup_{i=0}^{\infty} c_{2 i+1}\left(\left[0, t_{2 i+1}\right]\right), r^{-}\left(\mathbb{R}_{\geq}\right)\right) \leq \varepsilon .
\end{aligned}
$$

Since $t_{0}=1$ and $d\left(c_{0}\left(t_{0}\right), r^{+}\left(\mathbb{R}_{\geq}\right)\right) \leq \varepsilon$ we have $\measuredangle_{p}\left(\dot{c}_{0}(0), \dot{r}^{+}(0)\right) \leq \varepsilon$ and in the same way $\angle_{p}\left(\dot{c}_{1}(0), \dot{r}^{-}(0)\right) \leq \varepsilon$. Since $p \in F_{0}$ and $x_{\nu}=$ $c_{\nu}(\infty) \in F_{0}(\infty), \nu=0,1$, we have $\angle_{p}\left(\dot{c}_{0}(0), \dot{c}_{1}(0)\right)=\angle\left(x_{0}, x_{1}\right)$. Since 
$\varepsilon<(1 / 2) \angle\left(x_{0}, x_{1}\right)$ the rays $r^{+}$and $r^{-}$are distinct. Furthermore,

$$
\begin{aligned}
\angle\left(r^{+}(\infty), r^{-}(\infty)\right) & \geq \angle_{p}\left(\dot{r}^{+}(0), \dot{r}^{-}(0)\right) \\
& \geq \angle_{p}\left(\dot{c}_{0}(0), \dot{c}_{1}(0)\right)-2 \varepsilon=\angle\left(x_{0}, x_{1}\right)-2 \varepsilon .
\end{aligned}
$$

Since $x_{2 i} \rightarrow r^{+}(\infty)$ and $x_{2 i+1} \rightarrow r^{-}(\infty)$ in the sphere topology as $i \rightarrow \infty$ and $\angle\left(x_{i}, x_{i+1}\right)=\angle\left(x_{0}, x_{1}\right)$, it follows from the semicontinuity of $\angle$ that

$$
\angle\left(r^{+}(\infty), r^{-}(\infty)\right) \leq \angle\left(x_{0}, x_{1}\right) .
$$

In particular, $r^{+}(\infty)$ and $r^{-}(\infty)$ are in the same connected component.

We recall that $\Psi(p)=\{V \in \mathcal{V} \mid d(p, V) \leq a\}$. By (iii) and (iv) and since $\varepsilon<a / 2$ we see the existence of $\bar{t}_{i} \in[0, \infty)$ for $i=2,4,6, \ldots$ with $W_{i} \in \Psi\left(r^{+}\left(\bar{t}_{i}\right)\right)$. We want to show that $r^{+}(\infty)$ is in a nonstandard component. Arguing indirectly, there exists some $W \in \mathcal{W}$ with $W \in$ $\Psi \circ r^{+}(t)$ for each large enough $t$. This implies that $W \cap W_{2 k} \neq \varnothing$ for all large $k$. Thus we can find a configuration of four pairwise distinct $W_{1}^{\prime}, \ldots, W_{4}^{\prime} \in \mathcal{W}$, with $W_{\mu}^{\prime} \cap W_{\mu+1}^{\prime} \neq \varnothing$ and indices taken modulo 4 . Indeed, put $W_{1}^{\prime}:=W, W_{2}^{\prime}:=W_{2 k}, W_{3}^{\prime}:=W_{2 k+1}$ and $W_{4}^{\prime}:=W_{2 k+2}$ for some large $k$. An easy argument using the distance nonincreasing property of the geodesic projections onto higher rank subspaces shows the existence of a flat totally geodesic quatrilateral with a vertex in each $W_{\mu}^{\prime} \cap W_{\mu+1}^{\prime}$. By analyticity, this quatrilateral is contained in a flat intersecting $W_{\mu}^{\prime} \cap W_{\mu+1}^{\prime}$. This contradicts part (5) of Lemma 1.1. Hence $r^{+}(\infty)$ is not contained in a standard component.

To finish the proof it remains to construct the required situation which we do inductively. First we choose a point $p \in F_{0}$ and let $c_{0}, c_{1}:[0,1] \rightarrow F_{0}$ be the geodesics with $c_{0}(0)=c_{1}(0)=p$ into the direction of $x_{0}, x_{1}$, respectively. Then $d\left(c_{0}(1), W_{\nu}\right)=d\left(c_{1}(1), W_{\nu}\right)=0<\varepsilon$ for $\nu=0,1$. We now assume that we have already constructed $c_{0}, \ldots, c_{k}$ and $\sigma_{1}, \ldots, \sigma_{k-1}$ such that all the conditions (i) - (iv) are satisfied up to index $k$.

We now construct $\sigma_{k}$ and $c_{k+1}$. Note that by induction hypothesis $W_{k}$ is already defined. Let

$$
\Sigma_{k}:=\left\{\sigma \in \Sigma \mid \sigma W_{k}=W_{k} \text { and } \sigma x_{k}=x_{k}\right\} .
$$

Then $\Sigma_{k}$ operates with compact quotient on $W_{k}$ and by isometries on $\partial_{T} W_{k}$. Since $W_{k}$ splits as $Q_{k} \times \mathbb{R}$ and $Q_{k}$ has the visibility property, $\Sigma_{k}$ operates densely with respect to the sphere topology on the Tits geodesics of length $\pi$ connecting $x_{k}$ to the other singular point of $W_{k}(\infty)$. Thus 
there exists a sequence $\sigma_{k_{j}} \in \Sigma_{k}, j=1,2, \ldots$ such that $\sigma_{k_{j}}\left(x_{k-1}\right) \neq$ $x_{k-1}$ but $\sigma_{k_{j}}\left(x_{k-1}\right) \rightarrow x_{k-1}$ in the sphere topology as $j \rightarrow \infty$. We can also assume $\sigma_{k_{j}}\left(\boldsymbol{W}_{k-1}\right) \notin\left\{W_{1}, \ldots, W_{k}\right\}$. Let $\bar{c}_{k_{j}}$ : $\mathbb{R} \rightarrow X$ be the geodesic with $\bar{c}_{i_{j}}(0)=c_{k-1}\left(t_{k-1}\right)$ and $\bar{c}_{k_{j}}(\infty)=\sigma_{k_{j}}\left(x_{k-1}\right)$. We will finally choose $c_{k+1}:=\left.\bar{c}_{k_{j}}\right|_{\left[0, t_{k+1}\right]}$ for a suitable $j$ and $t_{k+1}$. By induction hypothesis we have

$$
\operatorname{Hd}\left(c_{*}\left(\left[0, t_{*}\right]\right) \cup \cdots \cup \bar{c}_{k-1}\left(\mathbb{R}_{\geq}\right), \overline{p x_{k-1}}\right)<\left(1-2^{-(k-1)}\right) \varepsilon .
$$

Since $\sigma_{k_{j}}\left(x_{k-1}\right) \rightarrow x_{k-1}$ as $j \rightarrow \infty$, it is obvious that for $j$ large enough

$$
\operatorname{Hd}\left(c_{*}\left(\left[0, t_{*}\right]\right) \cup \cdots \cup c_{k-1}\left(\left[0, t_{k-1}\right]\right) \cup \bar{c}_{k_{j}}\left(\mathbb{R}_{\geq}\right), \overline{p \sigma_{k_{j}}\left(x_{k-1}\right)}\right)<\left(1-2^{-(k+1)}\right) \varepsilon .
$$

Choose $\sigma_{k}:=\sigma_{k_{j}}$ for such $j$ large enough and $\bar{c}_{k+1}:=\bar{c}_{k_{j}}$.

Then (i), (ii) and (iv) are satisfied for $c_{0}, \ldots, c_{k},\left.\bar{c}_{k}\right|_{[0, t]}$ and $\sigma_{1}, \ldots, \sigma_{k}$ where $t>0$ is arbitrary.

It remains to prove the existence of $t_{k+1}$ to show (iii). Note that $\bar{c}_{k+1}(\infty)=x_{k+1}$ is a singular point in $W_{k+1}(\infty)$. We claim that

$$
\lim _{t \rightarrow \infty} d\left(\bar{c}_{k+1}(t), W_{k+1}\right)=0 .
$$

Otherwise, by Lemma 2.6 there would exist some $W \in \mathcal{W} \backslash\left\{W_{k+1}\right\}$ with $\lim _{t \rightarrow \infty} d\left(\bar{c}_{k+1}, W\right)=0$ and $x_{k+1}$ would be a singular point of $W(\infty)$. Furthermore, $W \cap W_{k+1} \neq \varnothing$. But the singular points of $W$ and $W_{k+1}$ have to be distinct. This proves the claim. It follows the existence of $t_{k+1}>0$ with $d\left(\bar{c}_{k+1}\left(t_{k+1}\right), W_{k+1}\right) \leq \varepsilon$. Then $c_{k}=\left.\bar{c}_{k+1}\right|_{\left[0, t_{k+1}\right]}$ is the required geodesic. q.e.d.

\section{An application to the structure of quasi-flats}

In this section we sketch an application of our results. As above, we consider a Riemannian universal covering space $X$ of a closed, realanalytic manifold of non positive curvature and dimension 4 . In addition we require that $X$ does not contain any 3 -flat.

We consider a special class of quasi-flats. Let $S^{1}$ be the standard circle of length $2 \pi$ and let $\alpha: S^{1} \rightarrow \partial_{T} X$ be a simple closed Tits geodesic

parametrized proportionally to arc length. Furthermore, let $o \in X$ be given. Then define a map

$$
f_{o, \alpha}: \mathbb{R}^{2} \rightarrow X
$$


in the following way. For $x \in \mathbb{R}^{2}$ with polar coordinates $(r, \theta) \in \mathbb{R}_{\geq} \times S^{1}$ let $f_{o, \alpha}(x)=\overline{o \alpha(\theta)}(r)$. We call such a map a conical quasi-flat. Indeed, $f_{o, \alpha}$ is a quasi-flat and this fact is not obvious. So we sketch the main arguments to prove this fact:

First one shows that $\alpha: S^{1} \rightarrow \partial_{T} X$ is a bilipschitz embedding. By Theorem 1 it is clear that $\alpha\left(S^{1}\right)$ is contained in a standard component. It follows that there are points $\theta_{1}<\theta_{2}<\cdots<\theta_{k}$ in $[0,2 \pi)$ which we view as points in $S^{1}$ and points $p_{i} \in X, i=1, \ldots, k$, such that

$$
\measuredangle_{p_{i}}\left(\alpha\left(\theta_{i-1}, \alpha\left(\theta_{i+1}\right)\right)=\angle\left(\alpha\left(\theta_{i-1}, \alpha\left(\theta_{i+1}\right)\right)<\pi\right.\right.
$$

for each $i$, where the indices are taken modulo $k$. Thus the geodesic rays from $p_{i}$ to the points in $\alpha\left(\left[\theta_{i-1}, \theta_{i+1}\right]\right)$ form an isometrically embedded flat sector $S_{i}$ which is obviously quasi-isometric to a sector of angle $d_{S^{1}}\left(\theta_{i-1}, \theta_{i+1}\right)$ in $\mathbb{R}^{2}$. Notice that $f_{o, \alpha}\left(\mathbb{R}^{2}\right)$ is in finite Hausdorff distance to $\bigcup_{i=1}^{k} S_{i}$. Now it is not difficult to prove that $f$ is a quasi-flat.

From our results in the previous section, we can deduce

Lemma 5.1. Suppose that $\alpha: S^{1} \rightarrow \partial_{T} X$ is a topologically embedded loop. Then $\alpha\left(S^{1}\right) \subset \partial_{T} X$ is the image of a simple closed Tits geodesic. Furthermore, the length spectrum of simple closed Tits geodesics in $\partial_{T} X$ is discrete.

Proof. Under the assumption of the lemma, $\alpha\left(S^{1}\right)$ is contained in a standard component of $\partial_{T} X$ by Theorem 1 . The standard components are described in Corollary 2.2. Since by assumption $X$ contains no $3-$ flats, this corollary implies the first part of the Lemma. To that end recall also the description of the Tits geometry of the elements in $\mathcal{W}$ in Section 1. By Lemma 1.1 and Lemma 2.7 the set $\left\{\operatorname{Td}\left(z_{1}, z_{2}\right) \mid z_{1}, z_{2} \in\right.$ $\left.\mathcal{R}_{3}(\infty)\right\}$ is discrete. This shows the final part of the lemma. q.e.d.

Now Theorem 2 is a consequence of B. Kleiner's results on the structure of maximal dimensional quasi-flats in cocompact Hadamard spaces (see $[6]$ ).

Proof of Theorem 2. Let $g: \mathbb{R}^{2} \rightarrow X$ be an $(L, C)$-quasi-flat. By [6], the limit set $g(\infty) \subset \partial_{T} X$ of the 2-dimensional quasi-flat is a 1-dimensional embedded sphere and its length is close to $2 \pi$ if $L$ is close to 1 . By Lemma 5.1 there exists a conical quasi-flat $f: \mathbb{R}^{2} \rightarrow X$ with $f(\infty)=g(\infty)$. Now Kleiner's results for quasi-flats in [6] imply that $g\left(\mathbb{R}^{2}\right)$ is in finite distance to $f\left(\mathbb{R}^{2}\right)$ and thus to a finite number of flats in $X$. By the discreteness of $\left\{\operatorname{Td}\left(z_{1}, z_{2}\right) \mid z_{1}, z_{2} \in \mathcal{R}_{3}(\infty)\right\}$ and by Lemma 5.1, we see that the number of these flats can be bounded in 
terms of the length of $g(\infty)$ and thus in terms of $L$. Moreover, $g(\infty)$ has length $2 \pi$ provided $L$ is sufficiently close to 1 . This completes the proof. q.e.d.

Proof of Corollary 3. We assume first that $X_{1}$ and $X_{2}$ do not contain isolated flats. The results of Section 2 show that the standard components of the Tits metric have the structure of a combinatorial graph. In this graph we can distinguish between two types of edges which we call short and long edges respectively. An edge is called long if it is contained in a cycle of combinatorial length 2 and short otherwise. The reader should observe that the long edges are exactly the edges of length $\pi$ in the Tits metric. The short edges have smaller Tits length. However, the crucial point is that this property of an edge can be read off purely from the combinatorial graph.

Each flat in $X_{1}$ or $X_{2}$ corresponds to a cycle in such a graph. The cycles which correspond to flats can also be detected completely using only the combinatorial data. These are precisely the following:

(i) The cycles of combinatorial length 2. The type of the sequence of edges is (long, long).

(ii) The cycles of length 3 . The type of the sequence of edges up to cyclic permutation is (long, short, short).

(iii) The cycles of length 4 with type (short, short, short, short).

Theorem 2 shows that a quasi-isometry $f: X_{1} \rightarrow X_{2}$ induces a map from cycles in the standard components of $\partial_{T} X_{1}$ to the cycles in the standard components of $\partial_{T} X_{2}$. Looking at finite intersections of conical quasiflats in $X_{1}$ and $X_{2}$ and at their images under $f$ and $f^{-1}$ one can show that $f$ induces a combinatorial map between the respective graphs. By the above it maps cycles corresponding to flats to cycles corresponding to flats and therefore $f$ maps flats to within finite distance of flats by Theorem 2. It is now clear that the Corollary also holds if $X_{1}$ or $X_{2}$ contains isolated 2-flats. q.e.d.

\section{References}

[1] U. Abresch \& V. Schroeder, Analytic manifolds of nonpositive curvature, Collection SMF Séminaires et Congrès 1 (ed. Arthur L. Besse), 1996.

[2] W. Ballmann, Lectures on spaces of nonpositive Curvature, DMV Seminar Band 25, Birkhäuser, Basel, 1995. 
[3] W. Ballmann, M. Gromov \& V. Schroeder, Manifolds of nonpositive curvature, PM 61, Birkhäuser, Boston, Basel, 1985.

[4] C. Croke \& B. Kleiner, Graphs of groups and ideal boundaries of Hadamard spaces, in preparation.

[5] A. Eskin \& B. Farb, Quasi-flats and rigidity in higher rank symmetric spaces, J. Amer. Math. Soc. 10 (1997) 653-692.

[6] B. Kleiner, Quasi-flats in Hadamard spaces, in preparation.

[7] B. Kleiner \& B. Leeb, Rigidity of quasi-isometries for symmetric spaces and Euclidean buildings, to appear in Inst. Hautes Études Sci. Publ. Math.

[8] V. Schroeder, Structure of flat subspaces in low-dimensional manifolds of nonpositive curvature, Manuscripta Math 64 (1989) 77-105.

University of PenNSylVANiA

UNIVERSITÄT ZÜRICH 